\title{
Awareness About Glaucoma Management Among Physicians in Riyadh: A Multicenter Cross- Sectional Study
}

Manal Alwazae ${ }^{1}$, Atheer Almutairi ${ }^{2}$, Atheer Alhumud 2 , Alhanouf Alhumidan ${ }^{2}$, Areej A. Alqahtani $\mathrm{II}^{3}$, Sumera Nisar ${ }^{4}$

1. Ophthalmology, College of Medicine, Princess Nourah Bint Abdulrahman University (PNU), Riyadh, SAU 2. Ophthalmology, Princess Nourah Bint Abdulrahman University (PNU), Riyadh, SAU 3. Pediatrics, College of Medicine, Princess Nourah Bint Abdulrahman University (PNU), Riyadh, SAU 4. Ophthalmology, Retina Consultant, Jeddah, SAU

Corresponding author: Atheer Almutairi, atheralmutiri@gmail.com

\section{Abstract \\ Purpose}

Glaucoma is an irreversible chronic disease that damages the optic nerve. Knowledge and skills related to glaucoma are extremely important for frontline physicians. This study aimed to determine the knowledge and management as well as examination and referral practices related to glaucoma among physicians at primary care centers and secondary hospitals in Riyadh, Saudi Arabia.

\section{Method}

This was a cross-sectional study of 126 physicians, including general practitioners as well as emergency, internal, and family physicians from three hospitals and five medical centers in Riyadh. A validated selfadministered questionnaire was used for data collection. It was divided into six categories: sociodemographic data and practice setting; and glaucoma assessment-questions regarding risk factors, knowledge, examination, management, and referral practices.

\section{Results}

Of the 126 participants, $32.8 \%$ were family physicians. Surprisingly, the overall knowledge score for glaucoma was suboptimal (34.2\%). While half of the doctors were aware of the medications used in glaucoma, $88.7 \%$ considered themselves unqualified to manage glaucoma. Although $93.7 \%$ agreed that increased ocular pressure requires urgent referral to an ophthalmologist, only $33.3 \%$ stated they were comfortable using tonometry.

\section{Conclusion}

Received 05/11/2020 Review began 05/18/2020 Review ended 05/28/2020 Published 06/05/2020

(c) Copyright 2020 Alwazae et al. This is an open access article distributed under the terms of the Creative Commons Attribution License CC-BY 4.0., which permits unrestricted use, distribution, and reproduction in any medium, provided the original author and source are credited.
The majority of physicians (65.8\%) showed a poor level of knowledge regarding glaucoma, which was reflected in their referral practices (66.9\%). Therefore, promoting increased glaucoma awareness along with improved examination skills and referral practice among frontline physicians is essential to prevent this avoidable cause of blindness.

Categories: Family/General Practice, Ophthalmology, Preventive Medicine Keywords: glaucoma practice, primary health care, blindness prevention

\section{Introduction}

Glaucoma is a condition that causes damage to the optic nerve, and it worsens over time if left untreated. It is often linked to a build-up of pressure inside the eye. Essentially, it is a chronic, irreversible disease that ultimately leads to optic neuropathy [1]. It is also a leading cause of blindness worldwide [2]. Globally, the prevalence of glaucoma is $3.54 \%$ in people above 40 years in age, which was estimated to be 64.3 million people in 2013, increasing to 76 million in 2020 [3]. In Saudi Arabia, the prevalence of blindness is $1.5 \%$, and glaucoma is responsible for $3 \%$ of all blindness in patients over 40 years old $[4,5]$. Therefore, glaucoma is considered to be one of the most common causes of blindness in Saudi Arabia.

A previous study has suggested that factors contributing to the development of glaucoma are age over 40 years, a family history of glaucoma, African race, and chronic diseases (e.g., diabetes mellitus, hypertension, and migraine) [6]. Due to its silent progressive nature, early detection through a full ocular examination is needed for at-risk patients. The full ocular examination includes measuring intraocular pressure (IOP), the visual field, and the cup-to-disc ratio. Thus, screening should assess all those aspects. One study failed to find a correlation between the development of glaucoma and high IOP levels alone [7]. However, the decision to seek medical care and early diagnosis may be altered by the absence of symptoms, which contributes to the fact that around half of patients are either undiagnosed or not treated in a timely manner 
$[8,9]$.

Interestingly, 50\% of diagnosed glaucoma cases present late and are revealed to have moderate to severe disease at initial diagnosis $[8,10,11]$. Moreover, managing patients with glaucoma can be challenging as it requires long-term follow-ups and adherence to the recommended treatment [12]. Therefore, the knowledge and skills of physicians in primary care centers and secondary hospitals regarding glaucoma are extremely important, as they are the frontline screeners in the healthcare system in Saudi Arabia; besides, they are the physicians closest to the patients [13]. In fact, referrals from family physicians account for $7 \%$ of the total glaucoma case referrals [8]. Hence, increasing physician awareness of glaucoma care practice could contribute greatly to preventing this leading cause of irreversible blindness [14].

To date, there is limited data in Saudi Arabia regarding the role of physicians at primary care centers and family physicians at secondary hospitals in glaucoma care. Hence, this study aimed to determine the knowledge and referral practices of physicians related to glaucoma in Riyadh, Saudi Arabia.

\section{Materials And Methods \\ Design, setting, and participants}

This cross-sectional study was conducted in three secondary hospitals and five primary care centers in Riyadh city, the capital of Saudi Arabia, from December 2018 to May 2019. We enrolled a total of 126 physicians, including general practitioners, family physicians, internists, and emergency medicine physicians. Family medicine physicians were enrolled from secondary hospitals and primary care centers. Of note, physicians at primary care centers in Saudi Arabia could be from diverse backgrounds as long as they have a diploma in family medicine. Therefore, internists, emergency medicine physicians, and general practitioners were recruited only from primary care centers, and they had the required credentials to work in primary care centers.

We calculated our sample size based on the expected level of physician awareness about glaucoma using the free $G *$ Power online software (release 3.1.9.2). Considering a level of glaucoma awareness of $70 \% \pm 13 \%$ and a $95 \%$ confidence interval (CI) at a level of significance $(\alpha=0.05)$ and a power of $80 \%(\beta=0.2)$, the estimated minimum sample size was 114 , which was increased to 126 to compensate for any incomplete data.

\section{Data collection}

A validated self-administered questionnaire was adapted from a Canadian study and used for data collection [8]. The questionnaire included six main sections: sociodemographic and practice setting, glaucoma risk factors, knowledge, examination, management, and referral. Sociodemographic data and practice setting included age, gender, position (resident, fellow, consultant), specialty, and frequency of seeing glaucoma patients. The questions assessed glaucoma in terms of risk factors (seven questions), knowledge (six questions), examination (four questions), management (six questions), and referral practices (seven questions). The responses were rated on a five-point Likert scale, ranging from 1 (strongly disagree) to 5 (strongly agree).

The total knowledge score was computed, and the variable was then transformed into a dichotomous measure. After consulting an expert in public health and epidemiology (Prof. Hala Elmorshedy), we defined adequate knowledge among participants as the ability to answer more than three questions ( $\geqslant 70 \%$ ); participants were deemed to have inadequate knowledge if they could answer only three or fewer questions $(\leqslant 50 \%)$. The same rule applied to all glaucoma assessment questions. Notably, all glaucoma assessment questions were based on the Canadian Ophthalmological Society evidence-based clinical practice guidelines for the management of glaucoma in adults [15].

\section{Data analysis}

The statistical analysis was conducted using SPSS Statistics version 23 (IBM Corp., Armonk, NY). The quantitative variables were described in means and standard deviations (SDs), and the qualitative variables were described in proportions. The association of glaucoma knowledge and referral practice was assessed with a chi-squared test, and p-values of $<0.05$ were considered statistically significant. We divided the Likert scale into two categories: agree (strongly agree, agree) and disagree (neutral, disagree, and strongly disagree).

\section{Ethical consideration}

The Institutional Review Board (IRB) approval number 18-0239 was obtained from the IRB Committee of Princess Nourah Bint Abdulrahman University. All participants provided verbal consent prior to enrollment.

\section{Results}

Table 1 presents the sociodemographic data and practice settings. A total of 126 physicians were involved in this study. They had a mean age of $37 \pm 8$ years; $58 \%$ were male and $32.8 \%$ were family physicians, and $44.3 \%$ 


\section{Cureus}

were residents in training. A minority of the physicians (36.6\%) reported that they saw patients with a diagnosis of glaucoma less than annually. The majority of physicians (77.9\%) reported that ophthalmologists provide the primary management for patients with glaucoma.

\begin{tabular}{|c|c|}
\hline Variables & Values \\
\hline Age in years, mean $\pm S D$ & $37 \pm 8$ \\
\hline Male, $\mathrm{n}(\%)$ & $76(58 \%)$ \\
\hline Female, n (\%) & $45(34.4 \%)$ \\
\hline \multicolumn{2}{|l|}{ Position: } \\
\hline Resident, $\mathbf{n}(\%)$ & $58(44.3 \%)$ \\
\hline Fellow, n (\%) & $15(11.5 \%)$ \\
\hline Consultant, n (\%) & $48(36.6 \%)$ \\
\hline \multicolumn{2}{|l|}{ Specialty: } \\
\hline General practitioner, n (\%) & $31(23.7 \%)$ \\
\hline Family physicians, $\mathrm{n}(\%)$ & $43(32.8 \%)$ \\
\hline Emergency medicine, $\mathrm{n}(\%)$ & $16(12.2 \%)$ \\
\hline Internal medicine, n (\%) & $36(27.5 \%)$ \\
\hline \multicolumn{2}{|l|}{ How often do you see glaucoma patients? } \\
\hline Daily, n (\%) & $2(1.5 \%)$ \\
\hline Weekly, n (\%) & $7(5.3 \%)$ \\
\hline Monthly, n (\%) & $21(16 \%)$ \\
\hline Semiannually, n (\%) & $36(27.5 \%)$ \\
\hline Annually, n (\%) & $12(9.2 \%)$ \\
\hline Less frequently, $\mathrm{n}(\%)$ & $48(36.6 \%)$ \\
\hline \multicolumn{2}{|c|}{ Who provides primary management to your patients who are diagnosed with glaucoma? } \\
\hline Primary care physicians, $\mathrm{n}(\%)$ & $5(3.8 \%)$ \\
\hline Ophthalmologist, $n$ (\%) & $102(77.9 \%)$ \\
\hline Optometrist, n (\%) & $4(3.1 \%)$ \\
\hline Ophthalmologist or optometrist, $\mathrm{n}(\%)$ & $10(7.6 \%)$ \\
\hline Not sure, but not the primary care physicians, $n(\%)$ & $5(3.8 \%)$ \\
\hline
\end{tabular}

\section{TABLE 1: Demographic data and practice setting}

SD: standard deviation

The majority of the physicians correctly agreed that the following items are risk factors for developing glaucoma: advancing age (85.6\%), steroid use (79.4\%), and family history (77.6\%). Regarding general knowledge about glaucoma, $93.7 \%$ of the physicians agreed that blindness could be prevented if glaucoma is detected and treated early, and 61.1\% recognized that early glaucoma does not present with central visual loss. However, most physicians did not consider themselves qualified to use tonometry (66.7\%), and only $48.4 \%$ were comfortable examining the optic nerve with a direct ophthalmoscope. Considering different modalities of glaucoma treatment, $56.3 \%$ of the doctors were aware of the medications used in glaucoma treatment. However, only around half of them were aware of the side effects of the medications. Most physicians (93.7\%) agreed that patients should be referred to an ophthalmologist if there was an increase in IOP. In comparison, only $71.2 \%$ agreed a referral was necessary if the patient's cup-to-disc ratio was greater than 0.5 (Table 2). 


\section{Cureus}

Questions

Agree, $\mathrm{n}(\%)$

Disagree, $\mathrm{n}(\%)$

Which of the following are risk factors for open-angle glaucoma? (Risk factors)

Advancing age

$107(85.6 \%)$

$18(14.4 \%)$

Family history

African descent

$97(77.6 \%)$

$28(22.4 \%)$

Chronic corticosteroid use

High blood pressure

Male gender

Last completed eye examination more than 5 years ago

$53(42.4 \%) \quad 72(57.6 \%)$

$100(79.4 \%) \quad 26(20.6 \%)$

$94(75.2 \%) \quad 31(24.8 \%)$

$64(51.2 \%) \quad 61(48.8 \%)$

$42(33.6 \%) \quad 83(66.4 \%)$

To what extent do you agree with each of the following statements? (Knowledge)

Blindness can be prevented if glaucoma is detected and treated early

$118(93.7 \%)$

$8(6.3 \%)$

Early open-angle glaucoma is symptomatic

$63(50 \%)$

$63(50 \%)$

Early glaucoma involves central visual field loss

$49(38.9 \%)$

$77(61.1 \%)$

Visual field loss can be detected after minor damage to nerve fibers

I am aware of the pathophysiology of glaucoma

$58(46 \%)$

$68(54 \%)$

87 (69\%)

$39(31 \%)$

I am familiar with the Canadian Ophthalmological Society guidelines for the management of glaucoma

$37(29.4 \%)$

$89(70.6 \%)$

To what extent do you agree with each of the following statements? (Examination)

I am comfortable performing tonometry

$42(33.3 \%)$

$84(66.7 \%)$

I am comfortable examining the optic nerve for glaucoma using the direct ophthalmoscope

$61(48.4 \%)$

$65(51.6 \%)$

I am comfortable assessing a visual field

$90(71.4 \%) \quad 36(28.6 \%)$

I am comfortable checking for a relative afferent pupillary defect

$79(62.7 \%)$

$47(37.3 \%)$

To what extent do you agree with each of the following statements? (Management)

I am comfortable managing general conjunctival and corneal diseases

$73(58.4 \%)$

$52(41.6 \%)$

I am comfortable managing optic nerve and retinal diseases

$40(31.7 \%)$

$86(68.3 \%)$

I am aware of the medications used in glaucoma

$71(56.3 \%)$

$55(43.7 \%)$

I am aware of the side effects of medications used in glaucoma

$67(53.6 \%)$

$58(46.4 \%)$

I am aware of the different lasers used in glaucoma

$41(32.5 \%) \quad 85(67.5 \%)$

I am aware of the different operations used in glaucoma

$47(37.3 \%) \quad 79(62.7 \%)$

To what extent do you agree with each of the following statements? (Referral)

Increased eye pressure

$118(93.7 \%)$

$8(6.3 \%)$

Peripheral visual field loss only

$107(84.9 \%) \quad 19(15.1 \%)$

Central visual field loss

$109(86.5 \%)$

$17(13.5 \%)$

Presence of risk factors for glaucoma

$92(74.2 \%) \quad 32(25.8 \%)$

Cup-to-disc ratio greater than 0.5

$89(71.2 \%) \quad 36(28.8 \%)$

Cup-to-disc ratio greater than 0.1 between the two eyes

$75(60 \%)$

$50(40 \%)$

$109(86.5 \%)$

TABLE 2: Glaucoma assessment questions 
Table 3 illustrates the participants' overall glaucoma knowledge, examination skills, management, and referral practices scores. The overall knowledge score was poor for $65.8 \%$ of the physicians. The majority of physicians (65.1\%) were not trained to perform ocular examinations, whereas $66 \%$ had good referral practice. Additionally, we did not find a significant association between glaucoma knowledge and referral practice $(\mathrm{p}=0.31)$. Moreover, we did not find a significant association between glaucoma knowledge and medical degree of participants $(\mathrm{p}=0.43)$ or their background $(\mathrm{p}=0.39)$.

\begin{tabular}{|c|c|c|}
\hline Overall scores & Good & Poor \\
\hline Knowledge & $34.2 \%$ & $65.8 \%$ \\
\hline Examination skills & $34.9 \%$ & $65.1 \%$ \\
\hline Management & $11.3 \%$ & $88.7 \%$ \\
\hline Referral practice & $66.9 \%$ & $33.1 \%$ \\
\hline
\end{tabular}

TABLE 3: Overall scores

\section{Discussion}

The physicians' scores on glaucoma knowledge, examination skills, and management were found to be low (34.2\%, 34.9\%, and $11.3 \%$, respectively). Furthermore, the majority had suboptimal referral practices (66.9\%). Although almost half of the participants were residents in training, and we did not find a significant association between glaucoma knowledge and medical degree $(\mathrm{p}=0.43)$. Unfortunately, this could affect the physician's role in the early detection of high-risk patients and timely referral to an ophthalmologist.

Looking specifically at the knowledge of glaucoma risk factors, most of the physicians agreed that family history, advancing age, and steroid use were strong risk factors for glaucoma development. Nevertheless, ophthalmologists expect primary care physicians to refer patients based mainly on risk factors rather than on examinations, which require special training and specific instruments [8]. Steroid use is considered a particularly important factor, and physicians at primary care centers should be aware of its role in the development of glaucoma. Physicians could acquire this information by merely performing histories and examinations and asking the patient about the use of any type of local or systemic steroid, including steroid injections for diabetic macular edema [16,17].

The general level of knowledge about glaucoma was poor among our respondents (34.2\%), and almost half of them were unaware of the side effects of glaucoma medications. Similarly, $46 \%$ of primary care physicians in Rotshtein et al.'s study reported that they received inadequate knowledge about glaucoma during their training, and 54\% were unfamiliar with the side effects of glaucoma medications [18]. Hence, the development of guidelines for glaucoma management targeting frontline physicians would help them to understand glaucoma medications and side effects so they can effectively counsel glaucoma patients.

Interestingly, the majority of the participants (93.7\%) agreed that they should refer patients with high IOP. However, only $33.3 \%$ of them were comfortable using tonometry. Similarly, a Canadian study reported that 97\% of family physicians would refer patients with high IOP, but only $30 \%$ were able to use tonometry [8]. Examination skills, including the use of tonometry and direct ophthalmoscopes, have been discussed thoroughly in the literature. Shuttleworth et al. found that only $56 \%$ of primary care practitioners were confident in performing direct ophthalmoscope examinations [19]. In Bell et al.'s study, only 9\% of glaucoma referrals came from general practitioners, and those that did were based on histories alone [20]. This highlights the need to train physicians at primary care centers to perform ocular examinations to detect patients needing referral to an ophthalmologist based on high IOP.

The results of our study could provide stakeholders and program directors of training programs with information regarding areas that need to be improved in the curriculum, with an emphasis on increasing knowledge about glaucoma management and risk factors and providing ocular examination training to support better referral practices. Nevertheless, physicians at primary care centers play a crucial rule in blindness prevention by ensuring that high-risk patients are screened and by supporting patients' optimal adherence to medical treatment [21-23].

\section{Limitations}

This study has some limitations. Our sample consisted solely of physicians and residents in training at primary care centers and secondary hospitals in Riyadh and the data cannot be generalized. 


\section{Conclusions}

This study revealed significant gaps in the knowledge about glaucoma among physicians at secondary hospitals and primary care centers in Riyadh, which was reflected in their referral practices. We believe that promoting increased glaucoma awareness and improved examination skills and referral practices among frontline physicians are essential to aid in the prevention of this leading cause of irreversible blindness.

\section{Additional Information \\ Disclosures}

Human subjects: Consent was obtained by all participants in this study. Institutional Review Board (IRB) Committee of Princess Nourah Bint Abdulrahman University issued approval 18-0239. The Institutional Review Board (IRB) approval number 18-0239 was obtained from the IRB Committee of Princess Nourah Bint Abdulrahman University. Participation was voluntary and all participants provided verbal consented prior to enrollment. Animal subjects: All authors have confirmed that this study did not involve animal subjects or tissue. Conflicts of interest: In compliance with the ICMJE uniform disclosure form, all authors declare the following: Payment/services info: All authors have declared that no financial support was received from any organization for the submitted work. Financial relationships: All authors have declared that they have no financial relationships at present or within the previous three years with any organizations that might have an interest in the submitted work. Other relationships: All authors have declared that there are no other relationships or activities that could appear to have influenced the submitted work.

\section{Acknowledgements}

We deeply appreciate Prof. Hala Elmoshedy for reviewing the method of this study. We also extend our gratitude to Hour Albalawi, Sarah Almari, Alanood Alsaleem, and Ghadeer Althaqib for collecting data for this study.

\section{References}

1. Owsley C, Rhodes LA, McGwin G Jr, et al.: Eye Care Quality and Accessibility Improvement in the Community (EQUALITY) for adults at risk for glaucoma: study rationale and design. Int J Equity Health. 2015, 14:135. 10.1186/s12939-015-0213-8

2. Pascolini D, Mariotti SP: Global estimates of visual impairment: 2010. Br J Ophthalmol. 2012, 96:614-618. 10.1136/bjophthalmol-2011-300539

3. Tham YC, Li X, Wong TY, Quigley HA, Aung T, Cheng CY: Global prevalence of glaucoma and projections of glaucoma burden through 2040: a systematic review and meta-analysis. Ophthalmology. 2014, 121:20812090. 10.1016/j.ophtha.2014.05.013

4. Al Obeidan SA, Dewedar A, Osman EA, Mousa A: The profile of glaucoma in a tertiary ophthalmic university center in Riyadh, Saudi Arabia. Saudi J Ophthalmol. 2011, 25:373-379. 10.1016/j.sjopt.2011.09.001

5. Tabbara KF, Ross-Degnan D: Blindness in Saudi Arabia. JAMA. 1986, 255:3378-3384. 10.1001/jama.1986.03370240048035

6. Hark L, Waisbourd M, Myers JS, et al.: Improving access to eye care among persons at high risk of glaucoma in Philadelphia--design and methodology: the Philadelphia Glaucoma Detection and Treatment Project. Ophthalmic Epidemiol. 2016, 23:122-130. 10.3109/09286586.2015.1099683

7. Chisholm IA: Management and diagnosis of glaucoma for the family physician . Can Fam Physician. 1990, 36:735-738.

8. Basilious A, Cheng J, Buys YM: Comparison of glaucoma knowledge and referral practices among family physicians with ophthalmologists' expectations. Can J Ophthalmol. 2015, 50:202-208. 10.1016/j.jcjo.2015.01.006

9. Tielsch JM, Sommer A, Katz J, Royall RM, Quigley HA, Javitt J: Racial variations in the prevalence of primary open-angle glaucoma: the Baltimore Eye Survey. JAMA. 1991, 266:369-374. 10.1001/jama.1991.03470030069026

10. Dandona L, Dandona R, Srinivas M, Mandal P, John RK, McCarty CA, Rao GN: Open-angle glaucoma in an urban population in southern India: the Andhra Pradesh Eye Disease Study. Ophthalmology. 2000, 107:1702-1709. 10.1016/s0161-6420(00)00275-X

11. Dandona L, Dandona R, Srinivas M, Mandal P, John RK, McCarty CA, Rao GN: Angle-closure glaucoma in an urban population in southern India. The Andhra Pradesh Eye Disease Study. Ophthalmology. 2000, 107:1710-1716. 10.1016/s0161-6420(00)00274-8

12. Prum BE Jr, Rosenberg LF, Gedde SJ, et al.: Primary Open-Angle Glaucoma Preferred Practice Pattern( $\left({ }^{\circledR}\right)$ guidelines. Ophthalmology. 2016, 123:P41-P111. 10.1016/j.ophtha.2015.10.053

13. Salikhova KM: The role of family doctor in early detection of glaucoma. (Article in Russian) . Probl Sotsialnoi Gig Zdravookhranenniiai Istor Med. 2020, 28:34-38. 10.32687/0869-866X-2020-28-1-34-38

14. Ichhpujani P, Bhartiya S, Kataria M, Topiwala P: Knowledge, attitudes and self-care practices associated with glaucoma among hospital personnel in a tertiary care center in North India. J Curr Glaucoma Pract. 2012, 6:108-112. 10.5005/jp-journals-10008-1116

15. Canadian Ophthalmological Society Glaucoma Clinical Practice Guideline Expert Committee: Canadian Ophthalmological Society evidence-based clinical practice guidelines for the management of glaucoma in the adult eye. Can J Ophthalmol. 2009, 44:S7-S54. 10.3129/109.080

16. Kersey J, Broadway D: Corticosteroid-induced glaucoma: a review of the literature . Eye (Lond). 2006, 20:407-416. 10.1038/sj.eye.6701895

17. Phulke S, Kaushik S, Kaur S, Pandav SS: Steroid-induced glaucoma: an avoidable irreversible blindness . J 


\section{Cureus}

Curr Glaucoma Pract. 2017, 11:67-72. 10.5005/jp-journals-10028-1226

18. Rotshtein A, Karkabi K, Geyer O, Cohen Castel OC: Primary care physicians' role perception and selfreported performance in glaucoma care: a survey study. BMC Res Notes. 2015, 8:776. 10.1186/s13104-0151770-z

19. Shuttleworth GN, Marsh GW: How effective is undergraduate and postgraduate teaching in ophthalmology? Eye (Lond). 1997, 11:744-750. 10.1038/eye.1997.189

20. Bell RW, O'Brien C: Accuracy of referral to a glaucoma clinic . Ophthalmic Physiol Opt. 1997, 17:7-11.

21. Berry LL, Parish JT, Janakiraman R, Ogburn-Russell L, Couchman GR, Rayburn WL, Grisel J: Patients' commitment to their primary physician and why it matters. Ann Fam Med. 2008, 6:6-13. 10.1370/afm.757

22. Rowe S, MacLean CH, Shekelle PG: Preventing visual loss from chronic eye disease in primary care: scientific review. JAMA. 2004, 291:1487-1495. 10.1001/jama.291.12.1487

23. Higginbotham EJ, Rust G: Ophthalmology and primary care: partners in peril . Arch Ophthalmol. 2008, 126:727-728. 10.1001/archopht.126.5.727 